\title{
El efecto del modelo docente y de la interacción con compañeros en las habilidades motrices creativas de la Danza. \\ Un formato de campo para su análisis y obtención de T-patterns motrices
}

\author{
The effect of teaching model and peer interaction in motor creative skills from Dance. \\ Afield format for analyze and getting motor T-patterns
}

\author{
*Carlota Torrents Martín, *Marta Castañer i Balcells, *Maria Dinušová y **M. Teresa Anguera \\ ${ }^{*}$ INEFC, Universitat de Lleida, ${ }^{* *}$ Universitat de Barcelona (España)
}

\begin{abstract}
Resumen: En el proceso de enseñanza-aprendizaje de disciplinas relacionadas con la Danza Contemporánea, la Danza creativa y de Improvisación así como la Expresión Corporal, la elección de la consigna y la forma de impartir ésta será fundamental para la consecución de los objetivos propuestos. El objetivo de este estudio será analizar el efecto de diferentes tipos de consignas para facilitar la generación divergente de respuestas con el fin de estimular la creatividad motriz. 12 sujetos sin experiencia previa en danza fueron observados durante 17 sesiones de iniciación a la Danza Contemporánea. La presencia de un modelo cinésico por parte del docente o la interacción con compañeros fueron aleatoriamente incluidos en las tareas. En el LOM -Laboratorio de Observación de la Motricidad- hemos elaborado un formato de campo ad hoc para la observación y se ha codificado con el programa Match Vision Studio (Perea, Alday y Castellano, 2004). Los datos y los T-Patterns obtenidos (Castañer, Torrents, Anguera y Dinušová, 2007) se analizaron mediante el programa Theme (Magnuson 1996) Los resultados muestran que los sujetos intentaron generar sus propias respuestas sin copiar el modelo propuesto por el docente pero que tendieron a copiar ciertas características de éste y que la divergencia en las respuestas se dio especialmente en las categorías de ritmo y dirección espacial.
\end{abstract}

Palabras clave: Danza Contemporánea, Creatividad motriz, Habilidad Motriz, Formato de Campo, T-Patterns motrices.

Abstract: The creative dancing, contact improvisation and body expression are academic areas where the decision about instructions and the teaching style will be relevant in order to get the aims. The proposal of this study will be to analyze the effect of different instruction kinds to manage the divergent getting answers, in order to stimulate the motor creativity. There were observed 12 participants that have not previous experience about dance, along 17 sessions about Contemporary Dance beginning. A kinesics teaching model or peer interaction has been randomly included in the tasks. In LOM -Laboratory of Human Motor Observation- we have built a field format ad hoc for the observation, and we have coded through Match Vision Studio (Perea, Alday y Castellano, 2004). The data have been analyzed through Theme (Magnusson, 1996), and we have obtained T-Patterns (Castañer, Torrents, Anguera \& Dinušová, 2007). The results show us that the participants have tried their own answers without to copy the model proposal by teacher, but they have tended to copy some traits of this, and the answers divergence has focused on rhythm and spatial direction categories. Key words: Contemporary Dance, motor creativity, motor skills, field format, motor T-patterns.

\section{Introducción}

Uno de los objetivos más importantes de disciplinas como la Danza Contemporánea, Creativa, o la Improvisación es la búsqueda de la expresión individual, así como el estímulo de la creatividad, la resolución de problemas y la expresión de sentimientos y pensamientos. Los alumnos se involucran física, emocional e intelectualmente utilizando diferentes técnicas de danza (Mac Donald, 1991, Lobo y Winsler, 2006). Así, se buscará el desarrollo de la creatividad motriz, que puede definirse como la combinación de percepciones para generar acciones motrices (Wyrick, 1968), y en la que influyen factores intelectuales, afectivos, culturales e incluso difiere con la edad. Para estimular la creatividad motriz en sesiones de danza, los docentes generalmente utilizan consignas abiertas, que pretenden generar respuestas singulares y frecuentemente improvisadas de los alumnos (Arteaga, 2003, Kalmar 2005, Ortiz, 2002, Stokoe, 1978). De hecho, la Improvisación se utiliza en otras disciplinas dancísticas como técnica para desarrollar el pensamiento divergente (Davenport, 2006). Estas consignas pueden presentarse mediante diferentes procedimientos docentes, como la adecuación de las condiciones de práctica, la utilización de feedbacks, el estímulo del pensamiento crítico, la muestra de las habilidades a ejecutar, o dando diferentes pautas (Riera, 2005). Durante las clases de danza, las condiciones de la práctica pueden modificarse fácilmente mediante la interacción con otros estudiantes o con la manipulación de objetos, y la

Fecha recepción: 17-12-07 - Fecha envío revisores: 28-12-07 - Fecha de aceptación: 17-01-08 Correspondencia: Carlota Torrents Martín

INEFC, Pda. de la Caparrella s/n

25192- Lleida (España)

E-mail: carlotat@inefc.es presentación de las tareas puede realizarse mediante la utilización de metáforas u otros recursos verbales. Por otro lado, la utilización de un modelo o ejemplo motriz dado por el mismo docente o por algún alumno no es tan común, ya que los docentes de este tipo de disciplinas basadas eminentemente en la creatividad no proponen modelos para copiar porque pretenden que los alumnos elaboren sus propias respuestas individuales (Stokoe, 1978).

No obstante, esta metodología didáctica no se basa en la literatura científica, ya que no hemos encontrado ninguna evidencia empírica que demuestre cual es la mejor forma de estimular la creatividad en la danza. ¿Es realmente decisivo que el docente no ofrezca ningún modelo como ejemplo? ¿Pueden los modelos ayudar a incrementar la producción divergente de respuestas? ¿Podemos utilizar otro tipo de consignas o podemos ofrecer otras pautas para motivar este pensamiento divergente? ¿Ayudará la interacción con compañeros o la manipulación de objetos?

Para valorar la creatividad nos hemos basado en las tres dimensiones tradicionales descritas por Guilford en 1950:

Fluidez: Número de respuestas. Generación de respuestas o soluciones dentro de una misma categoría.

Flexibilidad o variabilidad: Modificación o variación de las respuestas en función de las respuestas previas.

Originalidad: Respuestas inusuales o sorprendentes.

El objetivo de este estudio será el de analizar qué tipo de consignas pueden favorecer la producción divergente y creativa de respuestas en clases de danza con principiantes para poder introducir innovaciones metodológicas en los procesos de enseñanza-aprendizaje de estas disciplinas. Concretamente, estudiaremos como afecta la presentación de un modelo cinésico durante la descripción de la tarea (consigna) y la 


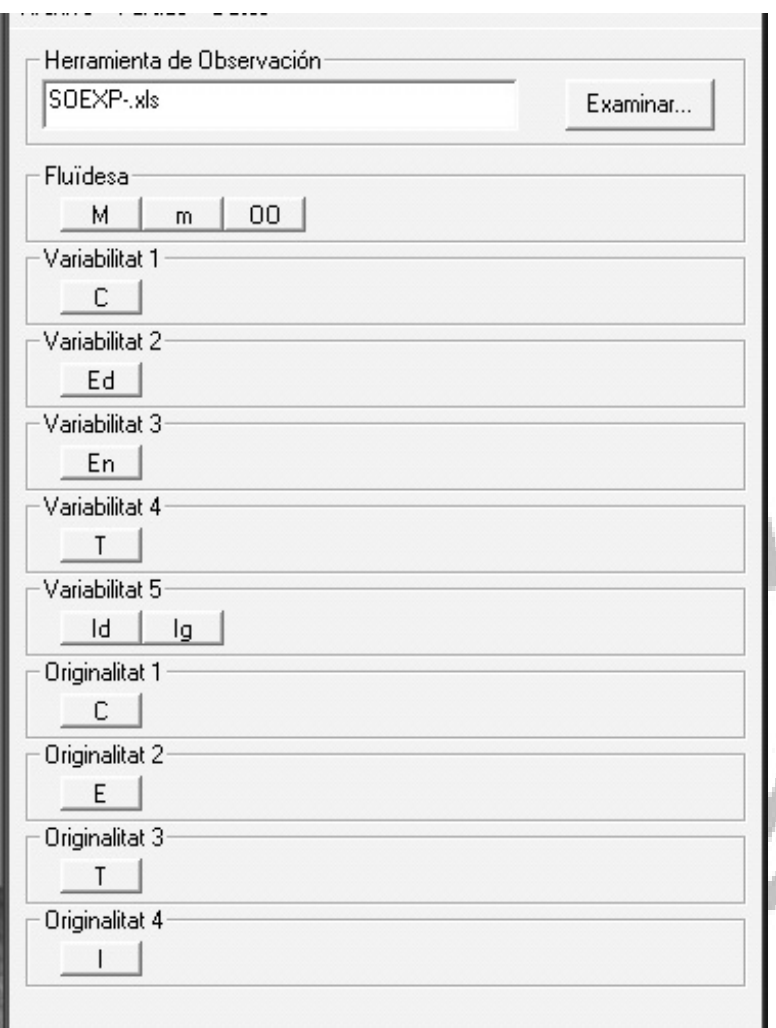

Figura 2. Pantalla del Match Vision Studio con el Formato de Campo

interacción con compañeros en la producción divergente de respuestas motrices. La complejidad y variabilidad de los contextos naturales requiere la elaboración de Sistemas de Categorías que nos permitan cuantificar la realidad. Una vez creado este sistema, se puede pasar a la evaluación de las respuestas de los alumnos.

\section{Método}

\subsection{Participantes}

Participaron en el estudio 120 estudiantes de segundo curso de la Licenciatura en Ciencias de la Actividad Física y el Deporte de la Universitat de Lleida que fueron divididos en 3 grupos de 40. Todos ellos cursaban la asignatura de Danza Contemporánea, basada principalmente en el trabajo de la técnica y la improvisación, y no tenían experiencia previa en este tipo de disciplina. Después de que dieran su consentimiento a participar en el estudio se seleccionaron aleatoriamente 4 participantes de cada grupo para el análisis de las respuestas, utilizando un total de 12 sujetos.

\subsection{Material}

Se elaboró un Formato de Campo ad hoc para analizar las habilidades motrices de manipulación y las de estabilidad que puede verse en la figura 2 y se describe a continuación. Para la codificación se ha utilizando el software Match Vision Studio (Perea Rodríguez y cols. 2004). Posteriormente, el software Theme (Magnusson, 1996) ha permitido la detección de patrones de análisis que nos muestran la relevancia y la configuración de los eventos registrados.

Para la confección de dicho Formato de Campo, se tuvieron en cuenta los criterios para analizar el movimiento expresivo descritos por Laban (Laban, 1991) y las tres dimensiones de la creatividad (fluidez, variabilidad y originalidad).

Para evaluar la primera dimensión o criterio (fluidez), se seleccionaron las siguientes categorías:

- Modelo exacto (mo): Respuesta igual al modelo propuesto por el docente
- Diferente al modelo (m): Respuestas diferentes al modelo propuesto por el docente

- Otras (a): Respuestas que no corresponden a la consigna propuesta

Para evaluar la segunda dimensión o criterio (variabilidad), se seleccionaron las siguientes categorías, relacionadas con la propuesta de Laban:

- Cambios corporales (c): Variaciones gestuales o posturales

- Cambio de dirección espacial (ed): Variaciones en la dirección espacial del movimiento

- Cambio de nivel espacial (in): Cambio entre los diferentes niveles del espacio (bajo o trabajo de suelo, medio o trabajo en bipedestación, alto o trabajo aéreo)

- Tiempo ( $\mathrm{t}$ ): Cambio de ritmo durante la ejecución de la acción

- Interacción en díada (id): Interacción con un compañero

- Interacción en grupo (ig): Interacción con más de un compañero

Para la evaluación de la originalidad, se atendió al criterio subjetivo de los observadores, y se seleccionaron las siguientes categorías:

- Movimientos inusuales o novedosos del cuerpo (c)

- Uso inusual o novedoso del espacio (e)

- Uso inusual o novedoso del ritmo $(\mathrm{t})$

- Interacción inusual o novedosa (i).

\subsection{Procedimiento}

Se ha utilizado la metodología observacional. Se filmaron 17 sesiones de 5' cada una después de un periodo de adaptación a la cámara para evitar el efecto de reactividad. Cada sesión se centró en la exploración motriz de un tipo de habilidades de estabilidad y de manipulación (Castañer y Camerino, 2006). Así 10 de ellas se focalizaron en tareas de estabilidad, diferenciadas en tareas de soporte, estabilidad axial y de detén. Las otras 7 focalizaron el trabajo en tareas de manipulación de un objeto, diferenciadas en habilidades de impacto o de conducción. En cada sesión, se combinaron aleatoriamente las variables de presencia o ausencia de modelo cinésico durante la presentación de la tarea y la interacción con compañeros mientras dichas tareas se llevaban a cabo (véase fig. 1). Todas las tareas se presentaron mediante consignas abier-

\begin{tabular}{|c|l|c|}
\hline \multicolumn{3}{|c|}{ Manipulación } \\
\hline \multirow{4}{*}{ Impacto } & Con modelo con compañero & $\mathrm{mmcc}$ cont \\
\cline { 2 - 3 } & Con modelo sin compañero & $\mathrm{mcc}$ cont \\
\cline { 2 - 3 } & Sin modelo sin compañero & $\mathrm{mc}$ cont \\
\hline \multirow{4}{*}{ Conducción } & Sin modelo sin compañero & $\mathrm{mc}$ \\
\cline { 2 - 3 } & Con modelo sin compañero & $\mathrm{mmc}$ \\
\cline { 2 - 3 } & Con modelo con compañero & $\mathrm{mmcc}$ \\
\hline & \multicolumn{2}{|c|}{ Estabilidad } \\
\hline \multirow{4}{*}{ Detén } & Sin modelo sin compañero & $\mathrm{mc}$ ax \\
\cline { 2 - 3 } & Con modelo sin compañero & $\mathrm{mmc}$ ax \\
\cline { 2 - 3 } & Con modelo con compañero & $\mathrm{mmcc}$ ax \\
\hline & Con modelo sin compañero & $\mathrm{mmc} \mathrm{de}$ \\
\cline { 2 - 3 } & Sin modelo con compañero & $\mathrm{mcc}$ de \\
\cline { 2 - 3 } & Con modelo con compañero & $\mathrm{mmcc}$ de \\
\hline \multirow{3}{*}{ Soporte } & Sin modelo con compañero & $\mathrm{mcc}$ so \\
\cline { 2 - 3 } & Con modelo con compañero & $\mathrm{mmcc}$ so \\
\cline { 2 - 3 } & Con modelo sin compañero & $\mathrm{mmc}$ so \\
\hline
\end{tabular}

Figura 1. Sesiones específicas de habilidades motrices con la aleatoriedad de la existencia o no de modelo y de interacción de grupo 

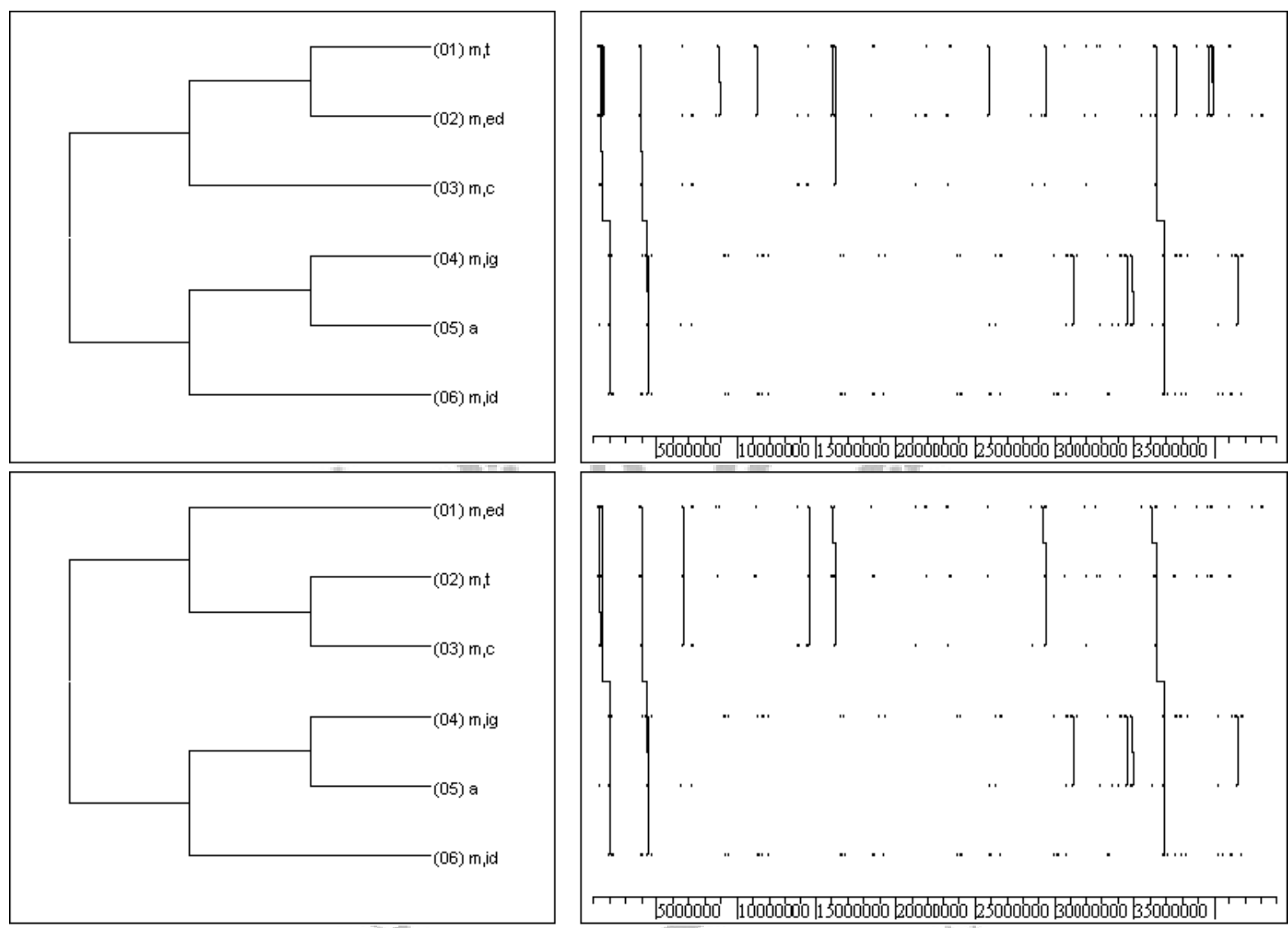

Figura 4. Primer y segundos T-Patterns significativos obtenidos del análisis de las sesiones de estabilidad

tas, proponiendo a los alumnos que explorasen las posibles respuestas dentro del criterio establecido por el docente.

\section{Resultados y discusión}

Tras el entrenamiento de los observadores la fiabilidad alcanzada ha sido de una concordancia del 95,30\% según el índice Kappa. Mediante elFormato de Campo construido se calcularon las tablas de frecuencias, que muestran el número de veces que se producía cada tipo de respuesta, así como los T-patterns significativos.

\subsection{Sesiones centradas en las habilidades de estabilidad}

Al observar la tabla de frecuencias, que se muestra en la figura 3, vemos que los resultados muestran como los sujetos, en las sesiones centradas en las habilidades de estabilidad, realizaron muchas más acciones motrices diferentes al modelo propuesto por el docente (m), que no

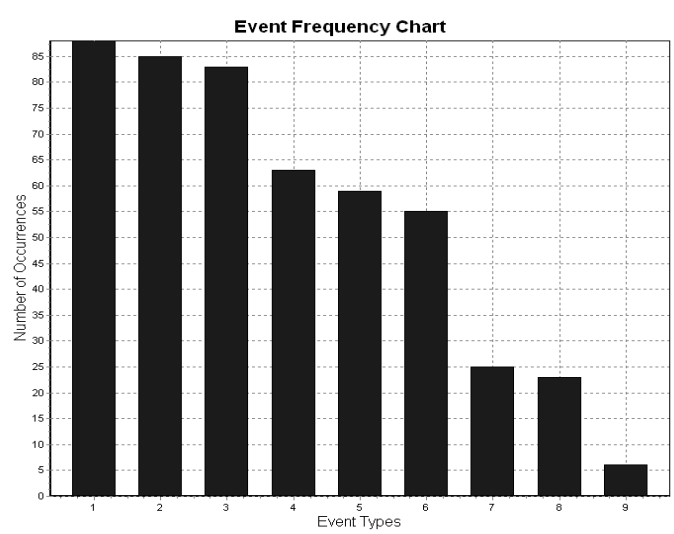

$1 \mathrm{~m} ; 2$ m,ig; 3 m,id; 4 mo; 5 m,ed; 6 m,t; 7 a; 8 m,c; 9 m,en

Figura 3. Tabla de Frecuencias de las habilidades de estabilidad acciones copiadas (mo). Seguramente, esto es debido a la inquietud de los alumnos por generar respuestas variadas y originales, ya que, a pesar de ser principiantes, conocían los fundamentos teóricos de este tipo de práctica y eran además sujetos con una gran experiencia motriz. No obstante, hay que destacar que, a pesar de esta premisa y de que aproximadamente en la mitad de las tareas no había modelo a copiar, el cuarto tipo de acción que más se da es precisamente la copia del modelo, lo que sugiere que este tipo de respuesta es también un recurso muy utilizado por los alumnos.

Las variaciones de las respuestas se dieron principalmente en el tipo de interacción (ig e id), y posteriormente en la dirección espacial (ed) y en el ritmo (t). La siguiente variación significativa se dio en la categoría de cuerpo (c) y la menos frecuente fue la de los cambios de nivel(en).

En cuanto a los T-Patterns, se obtuvieron 51 patrones significativos. Si analizamos los dos primeros (véase fig. 4) observamos que hay diferentes secuencias de acciones que se repiten con frecuencia. Concretamente, a menudo los cambios de dirección espacial vienen precedidos por cambios en el ritmo, y en ocasiones le sigue un cambio corporal. En el segundo patrón vemos como también ocurre a la inversa, que se dan cambios de ritmo precedidos de un cambio en la dirección espacial, para posteriormente también ejecutar un cambio corporal. Probablemente, esto es debido a que las habilidades de estabilidad están basadas en los impulsos, las paradas y los cambios de nivel, lo que fomentaba que se diesen cambios de ritmo y de dirección, en detrimento de los cambios en la postura o el gesto. En cuanto a la interacción, vemos que es frecuente que los alumnos empiecen trabajando en grupos, realicen acciones que no tienen que ver con la consigna y luego interactúen en parejas. Esto es debido a que los alumnos, una vez recibida la consigna, probaban alguna respuesta en grupo, comentaban lo sucedido o se organizaban, y empezaban a trabajar en parejas. Los resultados también nos muestran que, cuando el docente mostraba un modelo en pareja, los sujetos copiaban el tipo de interacción, pero ejecutaban acciones distintas. Es decir, se mantenían ciertos elementos del modelo pero se modificaban otros. 


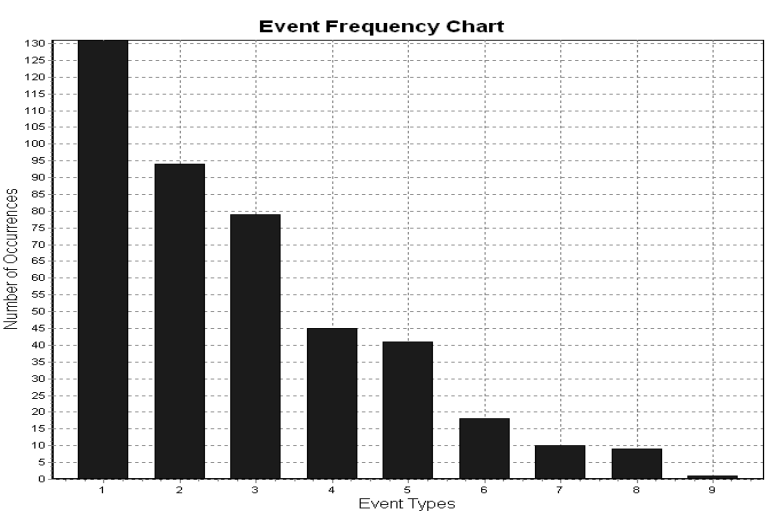

$1 \mathrm{~m} ; 2$ m,id; 3 m,t; 4 mo; 5 a; 6 m,ig; 7 m,ed; 8 m,c; 9 m,en

Figura 5. Tabla de frecuencias de las habilidades de manipulación

Las acciones que no tenían nada que ver con la consigna (a) sólo se produjeron en estas situaciones de interacción, probablemente para buscar el acuerdo entre el grupo, y al inicio de las sesiones, en las que a veces los alumnos mostraron una falta de atención o concentración.

\subsection{Sesiones centradas en las habilidades de manipulación}

La tabla de frecuencias de las habilidades de manipulación muestra resultados similares a la de las habilidades de estabilidad. Tal y como puede apreciarse en la figura 5, las respuestas diferentes al modelo son mucho más numerosas que las copias de éste. Aquí también vemos que los cambios en el tipo de interacción son los más frecuentes, aunque en este caso se relacionan más en pareja. Seguramente, el hecho de incluir un objeto hizo que los alumnos tuvieran una mayor inquietud por comunicarse con el compañero mediante el objeto. En cuanto al resto de variaciones, la que se dio con mayor frecuencia fue la de los cambios de ritmo. Las variaciones espaciales y corporales se dieron en pocas ocasiones, especialmente los cambios de nivel, que prácticamente fueron inexistentes. La inclusión de un objeto pudo influir en que los alumnos adoptasen posiciones y movimientos más similares al modelo que en las sesiones de estabilidad.

La poca presencia de cambios de nivel concuerda con la tendencia observada en sesiones de danza o Expresión Corporal con principiantes, en las que el docente tiene que insistir en la posibilidad de realizar cambios de nivel. En cuanto a la tendencia a modificar más aspectos temporales o espaciales que corporales, sugiere que los alumnos tienden a modificar ligeramente el modelo, pero no a proponer respuestas muy diferentes.

En cuanto a los T-Patterns, se obtuvieron 13 patrones significativos. Los dos primeros patrones significativos (véase fig. 6) muestran que a menudo la primera variación se da en la categoría de tiempo, coincidiendo con lo observado en la tabla de frecuencias. Muchos de ellos les siguen cambios de dirección, para posteriormente ejecutar acciones diferentes a la consigna (probablemente hablar con el compañero para llegar a algún acuerdo) y ponerse a trabajar en parejas. Así, volvemos a observar que las interacciones en díada se dan después de llegar a un acuerdo verbal con el compañero, en lugar de explorar directamente mediante el movimiento.

Este tipo de actuación, en el que los alumnos necesitan establecer un acuerdo verbal previo a las conductas en díada, no se hubiera producido si los alumnos hubieran tenido más experiencia en improvisación. Ésta se basa en el descubrimiento y experimentación de las posibilidades de movimiento en función de la situación que espontáneamente se genera, por lo que la comunicación con el compañero será un elemento más que incite a este descubrimiento.

Este estudio parece mostrar que la propuesta de un modelo cinésico por parte del docente o de algún compañero no estimula la copia exacta del modelo en un grupo de alumnos de este tipo, aunque también es un
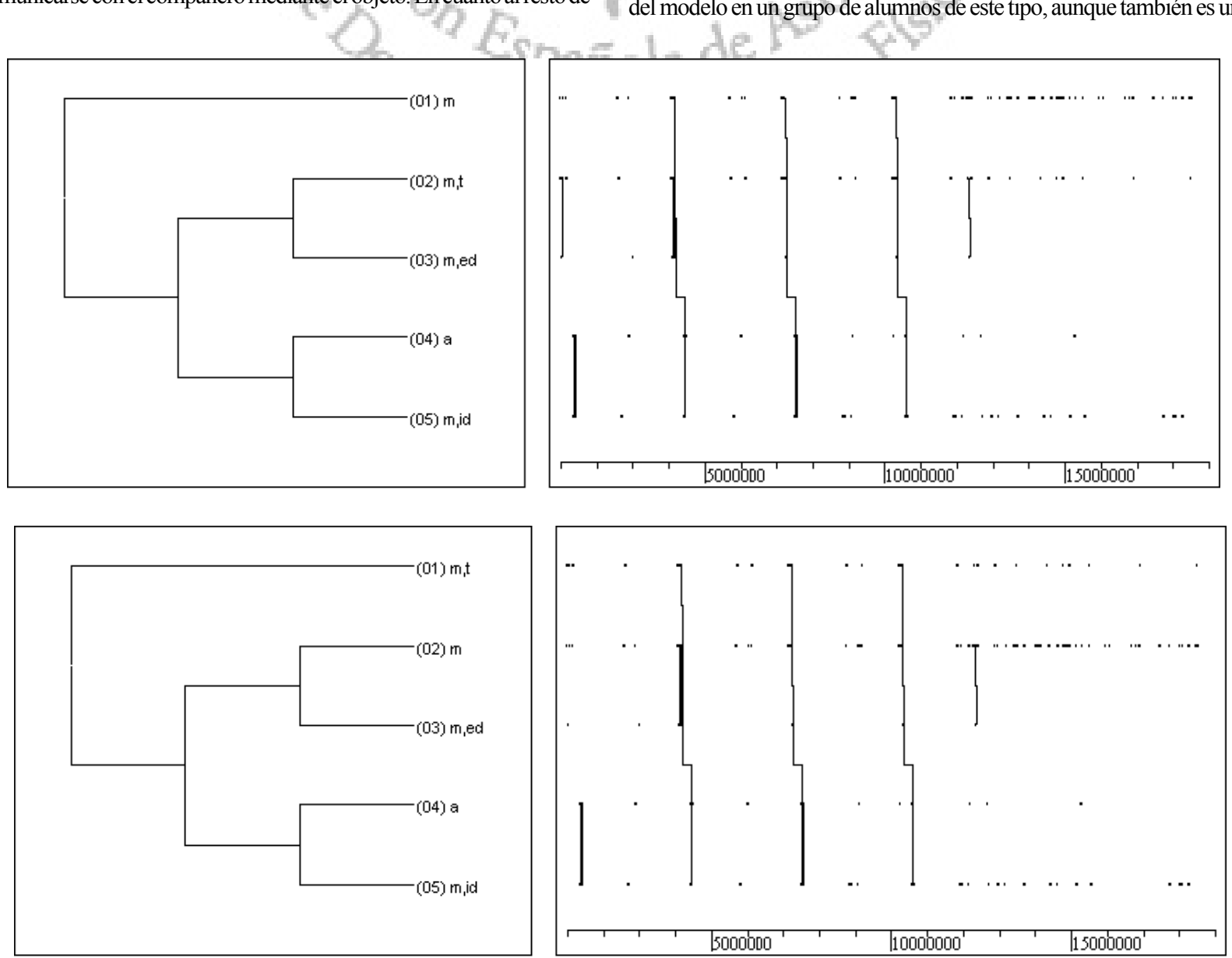

Figura 6. Primer y segundo T-Patterns significativos obtenido de las habilidades de manipulación 
recurso que se utiliza con frecuencia. Los alumnos intentan variar las propuestas y generar sus propias respuestas, pero copian algunas de las características principales del modelo. Por ejemplo, observamos que modifican el ritmo o la dirección especial, pero mantienen a menudo el tipo de movimiento o postura corporal adoptada para ejecutarlo. También copian el tipo de interacción, pero varían otros elementos de la tarea. Estos resultados sugieren que seria interesante que, en el criterio de fluidez, incluyéramos una nueva categoría de observación que incluyese aquellas respuestas que mantienen similitudes claras con el modelo pero no lo copian exactamente.

En cuanto a la interacción, parece ser que el trabajo en comunicación con otros compañeros (todos ellos principiantes en esta disciplina) genera un comportamiento diferente al trabajo individual, ya que los alumnos buscan un acuerdo verbal previo con sus compañeros.

El uso de objetos también parece afectar a la producción divergente de respuestas. Se produjeron más cambios de ritmo y espacio que corporales. A pesar de que los alumnos parecieran más motivados aexperimentar, probablemente los objetos dificultaban la tarea en cuanto a la atención que podían mantener al movimiento de su cuerpo.

Finalmente, cabe destacar que los observadores no registraron ninguna respuesta original. Esto seguramente es debido a la inexperiencia de los sujetos o al criterio demasiado exigente de los observadores. Así, esta categoría en este tipo de estudios requerirá otro tipo de herramientas para investigarla.

\section{Conclusiones}

El uso de consignas abiertas en sesiones de Danza Contemporánea focalizadas en la exploración del movimiento parece generar respuestas diferentes al modelo cinésico propuesto por el docente sobre principiantes en esta disciplina pero con formación deportiva. Estas diferencias se produjeron especialmente en los criterios relacionados con los cambios espaciales y temporales, y no tanto en la postura corporal o el gesto.

Las tareas que se ejecutaron con interacción con compañeros también fueron modificadas especialmente en cuanto al ritmo y la dirección espacial, pero mantuvieron el tipo de interacción propuesto por el modelo que dio el docente.

A pesar de que los alumnos modificaban los modelos, solían mantener las características más relevantes, por lo que se sugiere que, para futuras investigaciones, sería interesante incluir una nueva categoría que indicase aquellas respuestas similares al modelo pero no iguales.

Los observadores no registraron ninguna respuesta original, lo que sugiere que este criterio debería evaluarse mediante otros enfoques metodológicos puesto que la originalidad remite a criterios más encubiertos que netamente observables.

\section{Prospectiva}

Este estudio ha sido clave para optimizar otras investigaciones que estamos llevando a cabo en nuestro Laboratorio en que hemos establecido sistemas de categorías ad hoc para la observación de la Motricidad, la Expresión Corporal y la Danza Contact Improvisation (Torrents, Castañer y Dinušová, 2007). A partir de aquí estamos obteniendo $T$ Patterns significativos que nos aportan mucha información con relación a la práctica motriz de estas disciplinas.

Con todo ello y con lo que hemos expuesto en este artículo consideramos que hemos ofrecido algo de luz a las preguntas de investigación que hemos planteado al inicio y que volvemos a exponer ya que son preguntas clave para la optimización de muchos procesos de enseñanza-aprendizaje: ¿Pueden los modelos ayudar a incrementar la producción divergente de respuestas? ¿Podemos utilizar otro tipo de consignas o podemos ofrecer otras pautas para motivar este pensamiento divergente? ¿Ayudará la interacción con compañeros o la manipulación de objetos? Y, en definitiva, plantear si ¿Es realmente decisivo que el docente ofrezca o no modelos ejemplificadotes para el desarrollo de tareas motrices?

\section{Referencias}

Arteaga, M. (2003). Fundamentos de expresión corporal: Ámbito pedagógico. Granada: Universidad de Granada

Castañer, M. y Camerino, O. (2006). Manifestaciones Básicas de la Motricidad.Lleida: Universitat de Lleida-Inefc.

Castañer, M., Anguera, M.T., Torrents, C. \& Dinušová, M (2007): To identify and to analyze the motor answers in the corporal expression and Dance.Fifth Meeting of the European Research Group on Methodology for theAnalysis of Social Interaction, Eötvös University, Budapest.

Davenport, D. (2006). Building a dance composition course. An act of creativity. Journal of Dance Education 6(1), 25-32

Guilford, J.P. (1950). Creativity. American Psychologist, 5, 444-454.

Kalmar, D. (2005). Qué es la expresión corporal? Buenos Aires: Lumen

Laban, R. (1991). Danza educativa moderna. Barcelona: Paidós.

Magnusson, M. S. (1996). Hidden real-time patterns in intra- and interindividual behavior. European Journal of Psychological Assessment, 12 (2), 112-123.

Ortiz, M (2002). Expresión corporal: Una propuesta para el docenteado de educación fisica. Granada: Grupo Editor Universitario.

Perea Rodríguez, A., Alday Ezpeleta, L. y Castellano Paulis, J. (2004). Software para la observación deportiva match vision studio. III Congreso Vasco Del Deporte.

Riera, J. (2005). Las habilidades en el deporte. Barcelona: Inde publicaciones

Stokoe, P. (1978). Expresión corporal. Buenos Aires: Ricordi.

Torrents, Castañer y Dinušová (2007): Observation Category System of movement generation analysis in Contact Improvisation. 21th World Congress on Dance Research, 5-9 septiembre, Atenas.

Wyrick, W. (1968). The development of a test of motor creativity Research Quarterly, $n^{\circ}$ 39,3, pp.756-765.
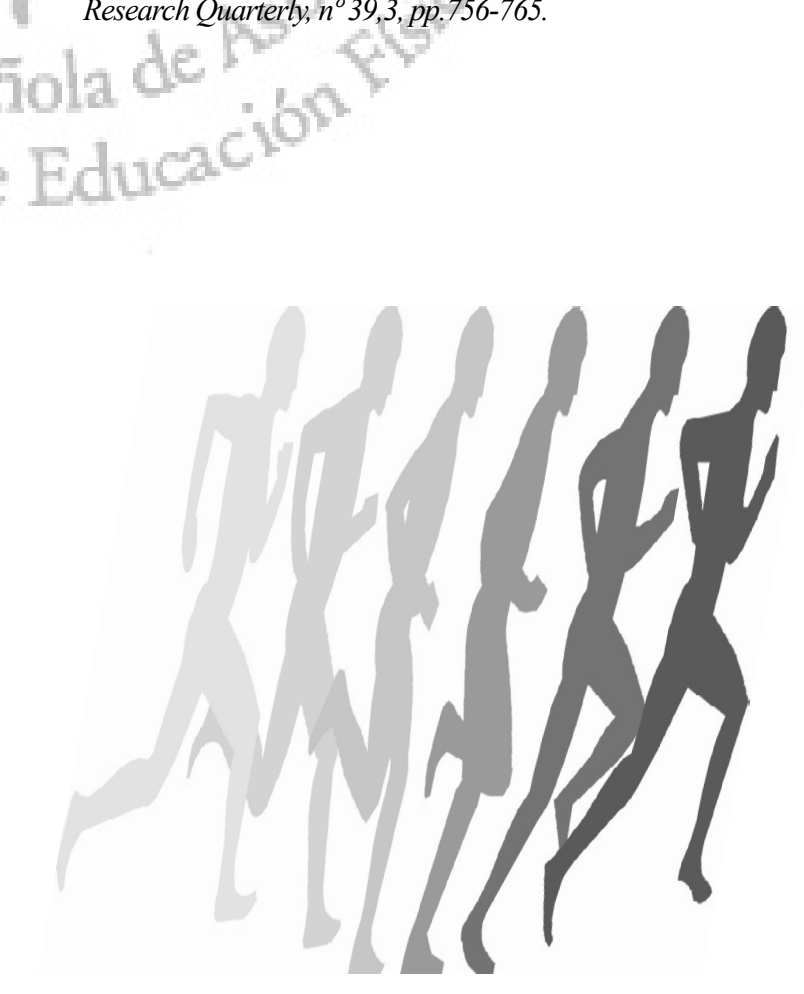